\title{
Effect of Long-term Exercise on Voiding Functions in Obese Elderly Women
}

\author{
Il Gyu Ko ${ }^{1,2}$, Mi Hee Lim², Pil Byung Choi ${ }^{3}$, Khae Hawn Kim4, Yong Seok Jee ${ }^{2}$ \\ ${ }^{1}$ Department of Physiology, Kyung Hee University School of Medicine, Seoul; \\ ${ }^{2}$ Department of Exercise Physiology and Prescription, Graduate School of Health Promotion, Hanseo University, Seosan; \\ ${ }^{3}$ Department of Leisure Sports and Recreation, Yeon Sung University, Anyang; \\ ${ }^{4}$ Department of Urology, Gachon University Gil Hospital, Gachon University of Medicine and Science, Incheon, Korea
}

\begin{abstract}
Purpose: An overactive bladder (OAB) may be defined as urgency that is a sudden, compelling, difficult to defer desire to pass urine that is usually accompanied by frequency and nocturia and possibly by incontinence. Obesity and old age are two factors in various causes of $\mathrm{OAB}$. Several epidemiologic studies have identified positive associations among obesity, old age, urinary incontinence, and OAB. However, although exercise has been known to improve obesity and reduce incontinent urine loss, little research has been done in elderly women. Therefore, we investigated the effects of exercise on obesity-related metabolic factors, blood lipid factors, and OAB symptoms in elderly Korean women.

Methods: Twenty-one women aged between 69 and 72 years were recruited from the Seoul senior towers in Korea. All subjects worked out on a motorized treadmill and stationary cycle for 40 minutes, respectively, and performed resistance exercise for 30 minutes once a day for 52 weeks. Body composition, blood pressure, blood lipids, OAB symptom score, and King's health questionnaire were investigated and analyzed.

Results: Before performing physical exercise, all subjects showed increased OAB symptoms in association with enhanced body mass index (BMI), percentage fat, and blood lipid profiles. However, physical exercise for 52 weeks suppressed BMI, percentage fat, and blood lipid profiles and thus improved OAB symptoms.

Conclusions: We suggest that long-term physical exercise can be a valuable tool for remarkable improvement of OAB.
\end{abstract}

Keywords: Overactive urinary bladder; Obesity; Exercise; Overactive bladder symptom score; King's health questionnaire

\section{INTRODUCTION}

Overactive bladder $(\mathrm{OAB})$ syndrome is characterized by urgency, usually with frequency and nocturia [1]. OAB is defined by the International Continence Society as urgency, with or without urgency incontinence, usually with increased daytime frequency and nocturia [2]. In general, frequency is usually defined as urinating more than 8 times per day.

Millions of women throughout the world are afflicted with $\mathrm{OAB}$, and interest in these symptoms has been growing in recent years as a consequence of the increased awareness of the human and social implications of $\mathrm{OAB}$ for the individual sufferer. $O A B$ represents a substantial problem in clinical practice, with an overall prevalence of $16 \%$ to $17 \%$ of adults. OAB is a symptom complex affecting both women and men. It is more common in the elderly, but young people have OAB symptoms as well [3]. Milsom et al. [3] reported that the prevalence of $\mathrm{OAB}$ increased from $3 \%$ in men aged 40 to 44 years to $42 \%$ in men older than 75 years. The cost of managing OAB in the United States has been estimated to be as high as 6 billion dollars [4].

The cause of $\mathrm{OAB}$ is widely recognized to be multifactorial, involving such areas as aging, urinary tract infection, urethral
Corresponding author: Yong Seok Jee

Department of Exercise Physiology and Prescription, Graduate School of Health Promotion, Hanseo University, 46 Hanseo 1-ro, Haemi-myeon, Seosan 356-706, Korea

Tel: +82-41-660-1028/Fax: +82-41-660-1088/E-mail: jeeys@hanseo.ac.kr

Submitted: August 16, 2013 / Accepted after revision: September 12, 2013
This is an Open Access article distributed under the terms of the Creative Commons Attribution Non-Commercial License (http://creativecommons.org/licenses/by-nc/3.0/) which permits unrestricted non-commercial use, distribution, and reproduction in any medium, provided the original work is properly cited. 
syndrome, pregnancy, diabetes mellitus, and obesity. Of these factors, obesity is an increasing health problem worldwide, and several epidemiologic studies have identified a positive association between obesity and an increased incidence of urinary incontinence and $\mathrm{OAB}$ [5-7]. Obesity occurs commonly in women with $\mathrm{OAB}$ and it has been proposed as a likely pathogenic process inviting neuronal, smooth muscle, and urethral dysfunction [8]. In women, the prevalence of OAB symptoms has been shown to be positively related to increasing body mass index (BMI) as shown by several authors [9-12]. Generally, BMI in ranges of 25 to $29.9 \mathrm{~kg} / \mathrm{m}^{2}$ and $\geq 30 \mathrm{~kg} / \mathrm{m}^{2}$, which correspond to overweight and obesity, respectively, has been shown to be an independent risk factor for $\mathrm{OAB}$ in women [13]. In the National Overactive Bladder Evaluation (NOBLE) Study, wet OAB was more prevalent in women with a BMI of 30 or more than in those with a BMI under $24 \mathrm{~kg} / \mathrm{m}^{2}$ [14].

Physical exercise including aerobic and resistance exercise is a common strategy in physical medicine and rehabilitation. Regular exercise is known to reduce the risk for cardiovascular disease and type II diabetes through numerous mechanisms. It reliably and robustly improves insulin sensitivity and cardiovascular fitness [15,16], reduces blood pressure [17], improves dyslipidemia [18], and improves both individual and combined factors of the metabolic syndrome score $[18,19]$. In addition, regular exercise has a modest effect on reducing body weight with substantially greater effects on improving body composition. Conversely, it is becoming increasingly clear that a continued sedentary lifestyle in overweight or obese individuals, particularly those who already have some metabolic abnormalities, comes at a high metabolic cost, as numerous health-related variables are worsened over relatively short time periods [20, 21].

In preventive treatments of $\mathrm{OAB}$ or urinary incontinence, pelvic muscle or aerobic exercise has been well-known to increase muscle strength and reduce incontinent urine loss. Furthermore, regular physical exercise is based on the idea of strengthening the muscles involved in keeping the urethra closed and the pelvic structures supported, particularly during periods of increased intra-abdominal pressure [22]. Although exercises are noninvasive, they may not be effective if there are variations in technique and compliance [23]. Therapeutic intervention, including pharmacologic agents, has been attempted to cure $\mathrm{OAB}$. However, medication therapy of $\mathrm{OAB}$ causes side effects, such as dry mouth, constipation, headache, and intestinal motility disorder.
Although the functional roles of physical exercise in obesity have been dramatically revealed, the effects of physical exercise in the relations of obesity and $\mathrm{OAB}$ in elderly women are still unknown. Therefore, in the present study we investigated the effects of long-term exercise on obesity-related factors (BMI, blood lipid profiles) and $\mathrm{OAB}$ symptoms in elderly women. For this study, body composition, blood pressure, blood lipids, the overactive bladder symptom score (OABSS), and scores on the King's health questionnaire (KHQ) were investigated and analyzed.

\section{MATERIALS AND METHODS}

\section{Subjects}

The thirty-five women aged between 69 and 72 years were recruited from the Seoul senior towers in Korea. The subjects were recruited on a voluntary basis from the Song-Do Hospital. The exclusion criteria were past or present neurological, hypertensive, or cardiopulmonary diseases. Persons $(\mathrm{n}=14)$ absent from 52 weeks of exercise five times or more per week were also excluded. Before the study, the principal investigator explained all the procedures to the subjects in detail. All subjects read and signed an informed consent form approved by the Ethical Committee of Hanseo University Institute and the Korean Academy of Medical Sciences before participation. Complete subject characteristics are listed in Table 1.

\section{Experimental Design}

On the first day, participants were required to read and sign an informed consent form and to complete a self-assessed questionnaire designed to identify subjects with OAB (OABSS and $\mathrm{KHQ}$ ). One day later, the subjects returned to the laboratory to complete baseline measurements, including body composition tests and blood lipid tests. The subjects in the exercise group

Table 1. Physical characteristics of the subjects $(n=21)$

\begin{tabular}{lr}
\hline Characteristic & Mean \pm SEM \\
\hline Age $(\mathrm{yr})$ & $71.76 \pm 11.08$ \\
Height $(\mathrm{cm})$ & $154.20 \pm 51.18$ \\
Weight $(\mathrm{kg})$ & $64.00 \pm 41.38$ \\
Body mass index $\left(\mathrm{kg} / \mathrm{m}^{2}\right)$ & $26.89 \pm 62.00$ \\
Lean body mass $(\mathrm{kg})$ & $45.05 \pm 53.06$ \\
Percent fat $(\%)$ & $29.40 \pm 92.55$ \\
\hline
\end{tabular}

SEM, standard error of the mean. 
performed the exercises on a motorized treadmill and stationary cycle for 40 minutes, respectively, and performed resistance exercise for 20 minutes once per day for 52 weeks. The followup testing included the same measures as in the baseline testing.

\section{Assessment of $O A B$}

All subjects completed $\mathrm{OAB}$ questionnaires to measure self-report (OABSS and KHQ) of function. The OABSS consists of a total of 4 questions regarding daytime frequency, nocturia, urgency, and urgency incontinence. The overall score is the sum of the 4 scores, and the diagnostic criteria for $\mathrm{OAB}$ are a total OABSS of 3 or more with an urgency score for Question 3 of 2 or more. In the event that the OABSS is used as the standard for the assessment of severity regarding $\mathrm{OAB}$, it is recommended that a total score of 5 or less be defined as mild, a score of 6 to 11 as moderate, and a score of 12 or more as severe. In this study, the OABSS was used as a reference [24]. Reliability of the OABSS was shown (Cronbach alpha $=0.523$ ).

The KHQ contains two single-item questions that address general health perceptions and the impact of incontinence. It also contains the following seven multiple item domains: role limitations, physical limitations, social limitations, personal relationships, emotions, sleep and energy, and severity (coping) measures. The minimum possible KHQ score is 0 (best health) and the maximum possible score is 100 (worst health) [25]. Reliability of the KHQ was shown (Cronbach alpha $=0.911$ ).

\section{Assessments of BMl and Body Composition}

The degree of obesity for all subjects was assessed by the BMI and body composition testing. For BMI, the subjects' heights were measured by using a wall-mounted ruler at the time of entry into the study. Body weights were measured by using a digital scale before breakfast and after the subjects had voided. BMI was calculated as weight in kilograms, divided by the height in meters squared. To measure the weight, fat mass, and muscle mass of the subjects, we used the bioelectrical impedance analysis method (InBody 320 Body Composition Analyzer; BioSpace, Seoul, Korea), and measured height by using a BMS 330 anthropometer (BioSpace) [26]. BMI and body composition were measured at baseline and during the last week of study.

\section{Blood Sampling}

The blood lipid profiles for all subjects were assessed by blood sampling. Blood samples were obtained in the morning after a 12-hour overnight fast. Venous blood samples were collected in ethylenediaminetetraacetic acid-coated tubes and were centrifuged at 3,000 rpm for 15 minutes at $4^{\circ} \mathrm{C}$. Plasma glucose was measured by a glucose oxidase method, and total cholesterol (TC), low-density lipoprotein cholesterol (LDL-C), high-density lipoprotein cholesterol (HDL-C), and triglyceride (TG) levels were measured by enzymatic procedures by use of an autoanalyzer (ABX Micros 60, Horiba, Irvine, CA, USA).

\section{Exercise Protocol}

The subjects in the exercise group took part in supervised progressive exercise training for 52 weeks (Table 2). During this study, all subjects agreed not to change their daily activity patterns, outside of their participation in this study. The subjects also agreed not to change their dietary habits throughout the study periods.

Exercise sessions were observed from morning to afternoon and lasted approximately 70 to 80 minutes. All subjects began the exercise session with a warm-up including gentle lower and upper extremity stretching for 10 minutes during the period of this study. This type of stretching for the elderly was meant to protect the whole body joints without hyper-flexion or hyperextension before the exercise session. This was followed by the aerobic portion of their training, which involved stationary cy-

Table 2. Exercise protocol

\begin{tabular}{|c|c|c|c|}
\hline Item & Exercise types & Intensity/time & Period \\
\hline $\begin{array}{l}\text { Warm } \\
\text { up }\end{array}$ & Stretching & Mild discomfort/10 min & $5 /$ wk \\
\hline \multirow{11}{*}{$\begin{array}{c}\text { Work } \\
\text { out }\end{array}$} & Treadmill walking & $50 \% \mathrm{VO}_{2}$ peak $/ 20 \mathrm{~min}$ & $5 /$ wk \\
\hline & Ergometer & $50 \% \mathrm{VO}_{2}$ peak $/ 20 \mathrm{~min}$ & $5 /$ wk \\
\hline & Resistance exercise $^{\text {a) }}$ & & \\
\hline & Leg press & $60 \%$ of $10 \mathrm{RM} \times 10$ reps $\times 2$ sets & $5 /$ wk \\
\hline & Shoulder press & $60 \%$ of $10 \mathrm{RM} \times 10$ reps $\times 2$ sets & $5 /$ wk \\
\hline & Leg extension & $60 \%$ of $10 \mathrm{RM} \times 10$ reps $\times 2$ sets & $5 /$ wk \\
\hline & Leg curl & $60 \%$ of $10 \mathrm{RM} \times 10$ reps $\times 2$ sets & $5 /$ wk \\
\hline & Arm curl & $60 \%$ of $10 \mathrm{RM} \times 10$ reps $\times 2$ sets & $5 /$ wk \\
\hline & Back extension & $60 \%$ of $10 \mathrm{RM} \times 10$ reps $\times 2$ sets & $5 /$ wk \\
\hline & Abdominal flexion & $60 \%$ of $10 \mathrm{RM} \times 10$ reps $\times 2$ sets & $5 /$ wk \\
\hline & Rotary torso & $60 \%$ of $10 \mathrm{RM} \times 10$ reps $\times 2$ sets & $5 /$ wk \\
\hline $\begin{array}{l}\text { Cool } \\
\text { down }\end{array}$ & Stretching & Mild discomfort/10 min & $5 /$ wk \\
\hline
\end{tabular}

$\mathrm{VO}_{2}$, volume oxygen; reps, repetitions.

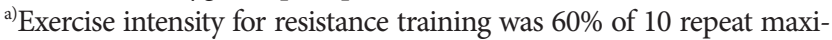
mum (RM) of maximal voluntary contraction test. 
cling and treadmill exercise for 40 minutes, respectively, at an intensity of approximately $50 \%$ of $\mathrm{VO}_{2}$ peak (peak oxygen volume) during this study. Resistance training was carried out by using weight-training machines. The exercise intensity for resistance training was $60 \%$ of the 10 repeat maximum of maximal voluntary contraction test. The order of resistance training was leg press, shoulder press, leg extension, leg curl, arm curl, back extension, abdominal flexion, and then rotary torso. Resistance training consisted of two sets. Also, the repetition of training initially consisted of 10 repetitions of each exercise.

\section{Data Analysis}

Data were analyzed by using IBM SPSS ver. 20.0 (IBM Co., Armonk, NY, USA). Data are expressed as means \pm standard errors of the mean (SEMs). For comparisons between the pre and post values, Wilcoxon's matched pairs test, a nonparametric statistical hypothesis test, was performed. Delta differences $(\Delta \%)$ in values between the pre and post measurements were calculated by using the following formula: $\Delta \%=$ (post-pre) $/$ pre $\times 100$. To analyze correlations among OABSS, KHQ, and body composition, Pearson test was used. The P-values less than 0.05 were taken as statistically significant.

\section{RESULTS}

\section{Effects of Long-term Exercise on OABSS in the Elderly Women}

The analysis of OABSS, including frequency, nocturia, urgency, and urinary incontinence, was performed in all subjects before and after regular physical exercise for 52 weeks, and the average values (mean $\pm S E M$ ) are shown in Table 3. First, the pretest results showed a moderate $\mathrm{OAB}$ score in all subjects. After 52 weeks, the scores for frequency, nocturia, urgency, and urinary incontinence were significantly decreased by physical exercise; in addition, the total score was significantly lowered. These results demonstrated that physical exercise for 52 weeks significantly decreased the OABSS in elderly women. In particular, among the four items, frequency and nocturia were the most changed in elderly women.

\section{Effects of Long-term Exercise on the KHQ in the Elderly Women}

The KHQ analysis, including general health, impact on life, role limitations, physical limitations, social limitations, relationships, emotions, sleep/energy, and incontinence, was performed in all subjects before and after regular physical exercise for 52 weeks. The average values (mean \pm SEM) are shown in Table 4 . First, the pretest results on the KHQ showed a "low-degree health condition" and a "moderate OAB" score in all of the subjects. After 52 weeks, the scores for general health, role limitations, physical limitations, relationships, emotions, sleep/energy, and incontinence were significantly decreased by physical exercise, whereas the impact on life and social limitation score were nonsignificant. These results showed that physical exercise for 52 weeks significantly improved general health, impact on life, role limitations, physical limitation, emotions, sleep/energy, and incontinence in elderly women. However, for the "impact on life" and "social limitation" items, physical exercise exerted no significant improvement.

\section{Effects of Long-term Exercise on Body Composition and Blood Pressure in the Elderly Women}

The body composition items including percentage fat, lean body mass (LBM), and BMI and the blood pressure items including systolic blood pressure (SBP) and diastolic blood pressure (DBP) were measured in all subjects before and after physical exercise for 52 weeks. The average values (mean \pm SEM) are shown in Table 5. The pretest results of body composition showed the de-

Table 3. Changes in OAB symptoms as assessed by the OABSS with exercise for 52 weeks

\begin{tabular}{lccccc}
\hline Item & Pre & Post & $\Delta \%$ & Z & P-value \\
\hline Frequency & $1.19 \pm 0.17$ & $0.66 \pm 0.10$ & $-35.00 \pm 8.19$ & 3.53 & 0.002 \\
Nocturia & $1.90 \pm 0.20$ & $1.33 \pm 0.73$ & $-27.78 \pm 6.73$ & 5.16 & 0.006 \\
Urgency & $2.66 \pm 0.21$ & $2.38 \pm 0.16$ & $-7.93 \pm 2.84$ & 2.82 & 0.010 \\
Urinary incontinence & $2.52 \pm 0.16$ & $2.28 \pm 0.17$ & $-8.33 \pm 4.87$ & 2.02 & 0.005 \\
Total score & $8.28 \pm 0.48$ & $6.66 \pm 0.29$ & $-16.42 \pm 4.02$ & 4.46 & 0.000 \\
\hline
\end{tabular}

Values are presented as mean \pm standard error of the mean.

$\mathrm{OAB}$, overactive bladder; OABSS, overactive bladder symptom score. 
Table 4. Changes in OAB symptoms as assessed by the KHQ with exercise for 52 weeks

\begin{tabular}{|c|c|c|c|c|c|}
\hline Item & Pre & Post & $\Delta \%$ & $\mathrm{z}$ & P-value \\
\hline General health & $48.81 \pm 5.31$ & $34.52 \pm 4.04$ & $-25.00 \pm 7.04$ & 3.873 & 0.001 \\
\hline Impact on life & $33.32 \pm 6.90$ & $28.57 \pm 5.77$ & $-7.93 \pm 5.09$ & 1.826 & 0.083 \\
\hline Role limitations & $34.92 \pm 5.26$ & $25.40 \pm 4.40$ & $-20.63 \pm 6.02$ & 3.875 & 0.001 \\
\hline Physical limitations & $36.51 \pm 5.59$ & $25.39 \pm 4.24$ & $-20.25 \pm 5.38$ & 3.569 & 0.002 \\
\hline Social limitations & $22.21 \pm 4.79$ & $18.25 \pm 3.80$ & $-11.10 \pm 4.50$ & 1.743 & 0.097 \\
\hline Emotions & $49.75 \pm 5.77$ & $40.74 \pm 4.43$ & $-12.14 \pm 4.09$ & 2.879 & 0.009 \\
\hline Sleep/energy & $53.97 \pm 5.97$ & $43.65 \pm 5.45$ & $-14.69 \pm 4.97$ & 2.914 & 0.009 \\
\hline Incontinence & $53.34 \pm 5.41$ & $46.99 \pm 4.28$ & $-8.33 \pm 2.82$ & 2.976 & 0.007 \\
\hline
\end{tabular}

Values are presented as mean \pm standard error of the mean.

$\mathrm{OAB}$, overactive bladder; KHQ, King's health questionnaire.

Table 5. Changes in body composition and blood pressure with exercise for 52 weeks

\begin{tabular}{|c|c|c|c|c|c|}
\hline Item & Pre & Post & $\Delta \%$ & $\mathrm{z}$ & P-value \\
\hline Body weight (kg) & $64.00 \pm 1.38$ & $61.32 \pm 1.51$ & $-10.33 \pm 0.88$ & 6.020 & 0.000 \\
\hline Percent fat $(\%)$ & $29.41 \pm 0.56$ & $26.00 \pm 0.65$ & $-11.72 \pm 0.91$ & 14.546 & 0.000 \\
\hline Lean body mass (kg) & $45.06 \pm 0.67$ & $46.31 \pm 0.59$ & $2.94 \pm 0.97$ & -3.080 & 0.006 \\
\hline Body mass index $\left(\mathrm{kg} / \mathrm{m}^{2}\right)$ & $26.89 \pm 0.44$ & $25.79 \pm 0.51$ & $-4.17 \pm 0.72$ & 6.124 & 0.000 \\
\hline Systolic blood pressure (mmHg) & $133.48 \pm 2.58$ & $126.43 \pm 2.62$ & $-5.03 \pm 1.71$ & 3.175 & 0.005 \\
\hline Diastolic blood pressure (mmHg) & $76.52 \pm 1.77$ & $73.43 \pm 2.82$ & $-3.61 \pm 3.69$ & 1.141 & 0.267 \\
\hline
\end{tabular}

Values are presented as mean \pm standard error of the mean.

Table 6. Changes in blood lipid factors with exercise for 52 weeks

\begin{tabular}{lccccc}
\hline Item & Pre & Post & $\Delta \%$ & Z & P-value \\
\hline Total cholesterol (mg/dL) & $225.90 \pm 7.65$ & $199.67 \pm 4.75$ & $-10.47 \pm 2.37$ & 3.945 & 0.001 \\
HDL-C (mg/dL) & $52.52 \pm 2.07$ & $56.29 \pm 2.41$ & $7.98 \pm 3.28$ & -2.706 & 0.014 \\
LDL-C (mg/dL) & $159.37 \pm 7.33$ & $124.78 \pm 4.99$ & $-19.98 \pm 3.05$ & 5.240 & 0.000 \\
Triglyceride (mg/dL) & $140.90 \pm 14.95$ & $108.52 \pm 10.79$ & $-20.45 \pm 4.11$ & 4.639 & 0.000 \\
\hline
\end{tabular}

Values are presented as mean \pm standard error of the mean.

HDL-C, high-density lipoprotein cholesterol; LDL-C, low-density lipoprotein cholesterol.

gree of obesity in the subjects. After 52 weeks, the analysis showed that average body weight, percentage fat, BMI, and SBP were significantly decreased by physical exercise. In addition, longterm physical exercise significantly enhanced LBM in the elderly women, whereas physical exercise exerted no significant effect on DBP. These results showed that physical exercise for 52 weeks significantly decreased percentage fat, BMI, and SBP in elderly women. Moreover, physical exercise significantly enhanced LBM in elderly women.

\section{Effects of Long-term Exercise on Blood Lipids in the Elderly Women}

The blood lipid variables including TC, LDL-C, HDL-C, and TG were measured in all subjects before and after physical exercise for 52 weeks. The average values (mean \pm SEM) are shown in Table 6. After 52 weeks, the analysis showed that average TC, LDL-C, and TG were significantly decreased by physical exercise. In addition, physical exercise significantly enhanced HDL$\mathrm{C}$ in the elderly women. These results showed that long-term physical exercise significantly decreased TC, LDL-C, and TG in 
Table 7. Relationships among variables of body composition, OABSS, and KHQ

\begin{tabular}{|c|c|c|c|c|c|c|c|c|c|c|c|c|c|c|}
\hline \multirow{2}{*}{ Item } & & \multicolumn{3}{|c|}{ Body composition } & \multirow{2}{*}{ OABSS $^{\text {a) }}$} & \multicolumn{9}{|c|}{ KHQ } \\
\hline & & $\%$ Fat & LBM & BMI & & $\mathrm{GH}$ & IL & $\mathrm{RL}$ & $\mathrm{PL}$ & SL & $\operatorname{Re}$ & Em & SE & IC \\
\hline \multirow[t]{2}{*}{$\%$ Fat } & $\mathrm{r}$ & 1.000 & -0.253 & 0.985 & -0.447 & -0.376 & -0.296 & -0.219 & 0.106 & 0.023 & - & -0.165 & 0.358 & -0.270 \\
\hline & $\mathrm{P}$-value & & 0.268 & 0.000 & 0.042 & 0.093 & 0.193 & 0.340 & 0.648 & 0.920 & & 0.474 & 0.111 & 0.236 \\
\hline \multirow[t]{2}{*}{ LBM } & $\mathrm{r}$ & & 1.000 & -0.265 & 0.158 & 0.411 & 0.185 & 0.192 & 0.425 & 0.185 & - & 0.306 & 0.217 & -0.391 \\
\hline & P-value & & & 0.246 & 0.494 & 0.064 & 0.423 & 0.405 & 0.055 & 0.421 & & 0.177 & 0.345 & 0.079 \\
\hline \multirow[t]{2}{*}{ BMI } & $\mathrm{r}$ & & & 1.000 & -0.465 & -0.386 & -0.257 & -0.276 & 0.096 & 0.045 & - & -0.193 & 0.354 & -0.232 \\
\hline & $\mathrm{P}$-value & & & & 0.034 & 0.084 & 0.261 & 0.226 & 0.680 & 0.845 & & 0.401 & 0.116 & 0.313 \\
\hline \multirow[t]{2}{*}{ OABSS } & $\mathrm{r}$ & & & & 1.000 & 0.046 & 0.160 & 0.260 & 0.284 & -0.051 & - & -0.260 & -0.042 & -0.034 \\
\hline & $\mathrm{P}$-value & & & & & 0.844 & 0.490 & 0.254 & 0.211 & 0.828 & & 0.254 & 0.856 & 0.885 \\
\hline \multirow[t]{2}{*}{$\mathrm{GH}$} & r & & & & & 1.000 & 0.111 & 0.285 & 0.127 & -0.167 & - & 0.528 & -0.003 & 0.091 \\
\hline & $\mathrm{P}$-value & & & & & & 0.633 & 0.210 & 0.584 & 0.470 & & 0.014 & 0.988 & 0.696 \\
\hline \multirow[t]{2}{*}{ IL } & $\mathrm{r}$ & & & & & & 1.000 & 0.357 & 0.333 & -0.192 & - & 0.126 & 0.110 & -0.103 \\
\hline & $\mathrm{P}$-value & & & & & & & 0.112 & 0.140 & 0.404 & & 0.586 & 0.635 & 0.658 \\
\hline \multirow[t]{2}{*}{$\mathrm{RL}$} & $\mathrm{r}$ & & & & & & & 1.000 & 0.218 & 0.005 & - & 0.050 & -0.038 & -0.021 \\
\hline & P-value & & & & & & & & 0.342 & 0.984 & & 0.829 & 0.869 & 0.927 \\
\hline \multirow[t]{2}{*}{ PL } & $\mathrm{r}$ & & & & & & & & 1.000 & 0.368 & - & 0.115 & 0.382 & -0.224 \\
\hline & $\mathrm{P}$-value & & & & & & & & & 0.101 & & 0.620 & 0.087 & 0.328 \\
\hline \multirow[t]{2}{*}{ SL } & $\mathrm{r}$ & & & & & & & & & 1.000 & - & 0.263 & -0.099 & -0.063 \\
\hline & $\mathrm{P}$-value & & & & & & & & & & & 0.250 & 0.670 & 0.786 \\
\hline \multirow[t]{2}{*}{$\operatorname{Re}$} & r & & & & & & & & & & 1.000 & - & - & - \\
\hline & P-value & & & & & & & & & & & & & \\
\hline \multirow[t]{2}{*}{ Em } & $\mathrm{r}$ & & & & & & & & & & & 1.000 & 0.073 & -0.028 \\
\hline & P-value & & & & & & & & & & & & 0.755 & 0.903 \\
\hline \multirow[t]{2}{*}{ SE } & $\mathrm{r}$ & & & & & & & & & & & & 1.000 & -0.118 \\
\hline & P-value & & & & & & & & & & & & & 0.611 \\
\hline \multirow[t]{2}{*}{ IC } & $\mathrm{r}$ & & & & & & & & & & & & & 1.000 \\
\hline & $\mathrm{P}$-value & & & & & & & & & & & & & \\
\hline
\end{tabular}

OABSS, overactive bladder symptom score; KHQ, King's health questionnaire; LBM, lean body mass; BMI, body mass index; GH, general health; IL, impact on life; RL, role limitations; PL, physical limitations; SL, social limitations; Re, relationships; Em, emotions; SE, sleep/energy; IC, incontinence.

${ }^{a)}$ Sum of 4 items in the OABSS.

elderly women. Moreover, physical exercise significantly enhanced HDL-C in elderly women.

\section{Correlations of the OABSS, KHQ, and Body Composition in the Elderly Women}

The relationships concerning $\Delta \%$ in body composition, OABSS, and KHQ are shown Table 7. Correlation analysis was performed to ascertain the relationships among the observed values. Most values except BMI and percentage fat did not show a significant correlation with OABSS or the general health and emotion vari- ables of the KHQ. On the other hand, there was a strong positive correlation between percentage fat and BMI and a weak negative correlation between percentage fat and OABSS. We also found a weak negative correlation between BMI and OABSS. Finally, there was a positive correlation between general health and emotion on the KHQ.

\section{DISCUSSION}

$\mathrm{OAB}$ comprises urinary urgency, with or without urgency in- 
continence, usually with frequency and nocturia in the absence of other pathologies. The incidence of OAB is higher in the elderly. Current population forecasts predict a worldwide increase in the proportion of people aged over 65 years, with the greatest rise being in those aged over 80 . With this in mind, it is reasonable to expect that the health care burden associated with $\mathrm{OAB}$ and lower urinary tract symptoms will also increase. In addition to this, the cause of $\mathrm{OAB}$ is widely recognized to be multifactorial and to include factors such as urinary tract infection, urethral syndrome, pregnancy, diabetes mellitus, and obesity.

Concerning obesity, BMI in the range of 25 to $29.9 \mathrm{~kg} / \mathrm{m}^{2}$ and $\geq 30 \mathrm{~kg} / \mathrm{m}^{2}$, which corresponds to overweight and obesity, respectively, has been shown to be an independent risk factor for $\mathrm{OAB}$ in women [8]. The present study showed the higher BMI of elderly women. This finding has been explained secondary to the changes in appetite, food intake, energy expenditure, and body composition that normally occur with aging, with an increase in fat mass and a decrease in muscle mass [27]. The results demonstrated that higher percentage fat in body composition was related to increasing age, whereas LBM decreased with aging in elderly women. These results suggest that aging caused increased body fat (\%) and BMI, whereas it decreased LBM, thus increasing obesity. In addition, the increase of BMI and percentage fat with aging caused elevated blood lipid factors such as TC, LDL-C, and TG in elderly women.

The exact mechanisms explaining the link between obesity and $\mathrm{OAB}$ are not well known, however. One hypothesis suggests that excess body weight increases intra-abdominal pressure, which in turn increases bladder pressure and intravesical pressure, thus leading to overactivity $[28,29]$. The previous study showed that a $B M I \geq 30 \mathrm{~kg} / \mathrm{m}^{2}$ was associated with a higher incidence of patient-reported urinary mixed leakage and pad use. Moreover, Hannestad et al. [13] reported a positive correlation between BMI and incontinence, and this association has been demonstrated for all subtypes of incontinence, particularly severe combined urge urinary and stress incontinence, and caused nocturia. Grady et al. [30] demonstrated that increasing BMI was a significant predictor of combined urge and stress incontinence. Because a higher BMI was age related, one may conclude that the above observation could be related to lower activity levels in older women. Another possibility is that the increased rate of urge incontinence in older women makes stress incontinence symptoms harder to isolate, which thus makes it more difficult to distinguish pure urge leakage from pure stress leakage or mixed leakage.
In this study, the $\mathrm{OAB}$ determination was based on both the OABSS and the KHQ, which is a validated questionnaire. The OABSS consists of a total of 4 questions regarding daytime frequency, nocturia, urgency, and urgency incontinence, and it is recommended that a total score of 5 or less be defined as mild, a score of 6 to 11 as moderate, and a score of 12 or more as severe. The KHQ contains two single-item questions that address general health perceptions and the impact of incontinence. It also contains the following seven multi-item domains: role limitations, physical limitations, social limitations, personal relationships, emotions, sleep/energy, and severity (coping) measures. The present results showed that higher scores on the OABSS and $\mathrm{KHQ}$ were related to increasing age. These results indicated that aging caused an increase in 4 items on the OABSS and enhanced 11 items of the KHQ, which thus signifies a "moderate" degree of $\mathrm{OAB}$.

Physical exercise has a modest effect on reducing body weight and blood lipid factors with substantially greater effects on improving body composition. In addition, regular exercise is known to increase muscle strength, including the rectus abdominis muscle, gluteus maximus, and pelvic muscle, and to reduce OAB symptoms. Above all, the effects of physical exercise induce weight loss. For this reason, regular physical exercise induced improvement in LUTS. The present results showed that 52 weeks of physical exercise significantly reduced BMI, percentage fat, and blood lipid factors (TC, LDL-C, TG) compared with before performing physical exercise. In addition, 52 weeks of physical exercise significantly enhanced LBM and HDL-C compared with before performing physical exercise. Our findings support previous reports indicating that physical exercise improves the imbalance in body composition and blood lipid factors that occurs with aging [31,32]. Generally, OAB patients showed a nonreflexive inhibition of involuntary detrusor contraction during the day and night. However, physical exercise may enable physical control of involuntary loss and a training of the will and the mind [33].

The present study showed that physical exercise during 52 weeks significantly decreased the OABSS in elderly women. Especially, the frequency and nocturia items of the OABSS were most suppressed in elderly women. In addition, 52 weeks of physical exercise improved the KHQ score compared with before performing physical exercise. Therefore, the results of the present study suggest that the beneficial effect of 52 weeks of physical exercise on $\mathrm{OAB}$ might change BMI and body composition. Moreover, the strengthening of muscles and tissues by 
physical exercise may relieve the symptoms of $\mathrm{OAB}$.

In this study, we evaluated the effect of 52 weeks of physical exercise on the aging-induced alteration of $\mathrm{OAB}$ symptoms, BMI, body composition, and blood lipid factors. All subjects before performing physical exercise showed an increase in $\mathrm{OAB}$ symptoms following enhanced BMI, percentage fat, and blood lipid factors (TC, LDL-C, TG). However, long-term physical exercise suppressed BMI, percentage fat, and blood lipid factors (TC, LDL-C, TG), thus improving OAB symptoms. We suggest that physical exercise can be a valuable tool for remarkable improvement of $\mathrm{OAB}$.

\section{CONFLICT OF INTEREST}

No potential conflict of interest relevant to this article was reported.

\section{ACKNOWLEDGEMENTS}

This research was supported by a 2012 grant from Hanseo University, Republic of Korea.

\section{REFERENCES}

1. Kim SE, Shin MS, Kim CJ, Park JH, Chung KJ, Jung H, et al. Effects of tamsulosin on urinary bladder function and neuronal activity in the voiding centers of rats with cyclophosphamide-induced overactive bladder. Int Neurourol J 2012;16:13-22.

2. Abrams P, Cardozo L, Fall M, Griffiths D, Rosier P, Ulmsten U, et al. The standardisation of terminology of lower urinary tract function: report from the Standardisation Sub-committee of the International Continence Society. Am J Obstet Gynecol 2002;187:116-26.

3. Milsom I, Abrams P, Cardozo L, Roberts RG, Thuroff J, Wein AJ. How widespread are the symptoms of an overactive bladder and how are they managed? A population-based prevalence study. BJU Int 2001;87:760-6.

4. Radomski SB, Barkin J. Medical management of overactive bladder. Can J Urol 2012;19 Suppl 1:2-9.

5. Cummings JM, Rodning CB. Urinary stress incontinence among obese women: review of pathophysiology therapy. Int Urogynecol J Pelvic Floor Dysfunct 2000;11:41-4.

6. Auwad W, Steggles P, Bombieri L, Waterfield M, Wilkin T, Freeman R. Moderate weight loss in obese women with urinary incontinence: a prospective longitudinal study. Int Urogynecol J Pelvic Floor Dysfunct 2008;19:1251-9.
7. Hunskaar S. A systematic review of overweight and obesity as risk factors and targets for clinical intervention for urinary incontinence in women. Neurourol Urodyn 2008;27:749-57.

8. Uzun H, Zorba OU. Metabolic syndrome in female patients with overactive bladder. Urology 2012;79:72-5.

9. Samsioe G, Heraib F, Lidfeldt J, Nerbrand C, Lindholm L, Agardh C, et al. Urogenital symptoms in women aged 50-59 years. Women's Health in Lund Area (WHILSA) Study Group. Gynecol Endocrinol 1999;13:113-7.

10. Schmidbauer J, Temml C, Schatzl G, Haidinger G, Madersbacher S. Risk factors for urinary incontinence in both sexes. Analysis of a health screening project. Eur Urol 2001;39:565-70.

11. Dallosso HM, McGrother CW, Matthews RJ, Donaldson MM; Leicestershire MRC Incontinence Study Group. The association of diet and other lifestyle factors with overactive bladder and stress incontinence: a longitudinal study in women. BJU Int 2003;92:69-77.

12. Dallosso HM, Matthews RJ, McGrother CW, Donaldson MM, Shaw C; Leicestershire MRC Incontinence Study Group. The association of diet and other lifestyle factors with the onset of overactive bladder: a longitudinal study in men. Public Health Nutr 2004;7: 885-91.

13. Hannestad YS, Rortveit G, Daltveit AK, Hunskaar S. Are smoking and other lifestyle factors associated with female urinary incontinence? The Norwegian EPINCONT Study. BJOG 2003;110:247-54.

14. Stewart WF, Van Rooyen JB, Cundiff GW, Abrams P, Herzog AR, Corey R, et al. Prevalence and burden of overactive bladder in the United States. World J Urol 2003;20:327-36.

15. Duscha BD, Slentz CA, Johnson JL, Houmard JA, Bensimhon DR, Knetzger KJ, et al. Effects of exercise training amount and intensity on peak oxygen consumption in middle-age men and women at risk for cardiovascular disease. Chest 2005;128:2788-93.

16. Church TS, Earnest CP, Skinner JS, Blair SN. Effects of different doses of physical activity on cardiorespiratory fitness among sedentary, overweight or obese postmenopausal women with elevated blood pressure: a randomized controlled trial. JAMA 2007;297: 2081-91.

17. Kelley GA, Sharpe Kelley K. Aerobic exercise and resting blood pressure in older adults: a meta-analytic review of randomized controlled trials. J Gerontol A Biol Sci Med Sci 2001;56:M298-303.

18. Kraus W, Slentz C. Metabolic syndrome: recognition, etiology and physical fitness as a component. In: Opara E, editor. Nutrition \& diabetes: pathophysiology and management. New York: Taylor \& Francis; 2006. p. 57-78.

19. Johnson JL, Slentz CA, Houmard JA, Samsa GP, Duscha BD, Aiken LB, et al. Exercise training amount and intensity effects on meta- 
bolic syndrome (from Studies of a Targeted Risk Reduction Intervention through Defined Exercise). Am J Cardiol 2007 15;100: 1759-66.

20. de Groot PC, Bleeker MW, Hopman MT. Magnitude and time course of arterial vascular adaptations to inactivity in humans. Exerc Sport Sci Rev 2006;34:65-71.

21. Hamilton MT, Hamilton DG, Zderic TW. Role of low energy expenditure and sitting in obesity, metabolic syndrome, type 2 diabetes, and cardiovascular disease. Diabetes 2007;56:2655-67.

22. Ko IG, Kim SE, Kim BK, Shin MS, Kim CJ, Yim SJ, et al. Swimming: effects on stress urinary incontinence and the expression of nerve growth factor in rats following transabdominal urethrolysis. Int Neurourol J 2011;15:74-81.

23. Aukee P, Immonen P, Laaksonen DE, Laippala P, Penttinen J, Airaksinen $\mathrm{O}$. The effect of home biofeedback training on stress incontinence. Acta Obstet Gynecol Scand 2004;83:973-7.

24. Jeong SJ, Homma Y, Oh SJ. Korean version of the overactive bladder symptom score questionnaire: translation and linguistic validation. Int Neurourol J 2011;15:135-42.

25. Kelleher CJ, Cardozo LD, Khullar V, Salvatore S. A new questionnaire to assess the quality of life of urinary incontinent women. $\mathrm{Br}$ J Obstet Gynaecol 1997;104:1374-9.

26. Lee KY, Lee HJ, Kim SE, Choi PB, Song SH, Jee YS. Short term rehabilitation and ankle instability. Int J Sports Med 2012;33:485-96.
27. Chapman IM. Obesity paradox during aging. Interdiscip Top Gerontol 2010;37:20-36.

28. Noblett KL, Jensen JK, Ostergard DR. The relationship of body mass index to intra-abdominal pressure as measured by multichannel cystometry. Int Urogynecol J Pelvic Floor Dysfunct 1997; 8:323-6.

29. Chancellor MB, Oefelein MG, Vasavada S. Obesity is associated with a more severe overactive bladder disease state that is effectively treated with once-daily administration of trospium chloride extended release. Neurourol Urodyn 2010;29:551-4.

30. Grady D, Brown JS, Vittinghoff E, Applegate W, Varner E, Snyder T, et al. Postmenopausal hormones and incontinence: the Heart and Estrogen/Progestin Replacement Study. Obstet Gynecol 2001;97: 116-20.

31. Soto PF, Herrero P, Schechtman KB, Waggoner AD, Baumstark JM, Ehsani AA, et al. Exercise training impacts the myocardial metabolism of older individuals in a gender-specific manner. Am J Physiol Heart Circ Physiol 2008;295:H842-50.

32. Nicklas BJ, Wang X, You T, Lyles MF, Demons J, Easter L, et al. Effect of exercise intensity on abdominal fat loss during calorie restriction in overweight and obese postmenopausal women: a randomized, controlled trial. Am J Clin Nutr 2009;89:1043-52.

33. Bo K, Berghmans LC. Nonpharmacologic treatments for overactive bladder-pelvic floor exercises. Urology 2000;55(5A Suppl):7-11. 